\title{
Study of the Convergence of the Increments of Gaussian Process
}

\author{
Abdelkader Bahram1, Shaban A. El-Shehawy ${ }^{2}$ \\ ${ }^{1}$ Department of Mathematics, Djillali Liabes University, Sidi Bel Abbès, Algeria \\ ${ }^{2}$ Department of Mathematics, Faculty of Science, Menoufia University, Shebin El-Kom, Egypt \\ Email: menaouar 1926@yahoo.fr, shshehawy64@yahoo.com
}

Received 29 April 2015; accepted 30 May 2015; published 2 June 2015

Copyright (C) 2015 by authors and Scientific Research Publishing Inc.

This work is licensed under the Creative Commons Attribution International License (CC BY).

http://creativecommons.org/licenses/by/4.0/

(c) (i) Open Access

\section{Abstract}

Let $\{X(t) ; t \geq 0\}$ be a Gaussian process with stationary increments $E\{X(t+s)-X(t)\}^{2}=\sigma^{2}(s)$. Let $a_{t}(t \geq 0)$ be a nondecreasing function of $t$ with $0 \leq a_{t} \leq t$. This paper aims to study the almost sure behaviour of $\limsup _{k \rightarrow \infty} \sup _{0 \leq s \leq a_{t_{k}}} \beta_{\left(t_{k}, \alpha\right)}\left|X\left(t_{k}+s\right)-X\left(t_{k}\right)\right|$ where

$$
\beta_{\left(t_{k}, \alpha\right)}=\left[2 \sigma^{2}\left(a_{t_{k}}\right)\left(\log \left(t_{k} / a_{t_{k}}\right)+\alpha \log \log t_{k}+(1-\alpha) \log \log a_{t_{k}}\right)\right]^{-1 / 2}
$$

with $0 \leq \alpha \leq 1$ and $\left\{t_{k}\right\}$ is an increasing sequence diverging to $\infty$.

\section{Keywords}

Wiener Process, Gaussian Process, Law of the Iterated Logarithm, Regularly Varying Function

\section{Introduction}

Let $\{W(t) ; t \geq 0\}$ be a standard Wiener process. Suppose that $a_{t}(t \geq 0)$ is a nondecreasing function of $t$ such that $0<a_{t} \leq t$ with $a_{t} / t$ is nonincreasing and $\left\{t_{k}\right\}$ is an increasing sequence diverging to $\infty$. In [1] the following results are established.

i) If $\limsup _{k \rightarrow \infty}\left(t_{k+1}-t_{k}\right) / a_{t_{k}}<1$, then

$$
\limsup _{k \rightarrow \infty} \sup _{0 \leq s \leq a_{t_{k}}} \lambda_{\left(t_{k}, \alpha\right)}\left|W\left(t_{k}+s\right)-W\left(t_{k}\right)\right|=1 \quad \text { a.s. }
$$

and 


$$
\limsup _{k \rightarrow \infty} \lambda_{\left(t_{k}, \alpha\right)}\left|W\left(t_{k}+a_{t_{k}}\right)-W\left(t_{k}\right)\right|=1 \quad \text { a.s. }
$$

where $0 \leq \alpha \leq 1$ and

$$
\lambda_{\left(t_{k}, \alpha\right)}=\left[2 a_{t_{k}}\left(\log \left(t_{k} / a_{t_{k}}\right)+\alpha \log \log t_{k}+(1-\alpha) \log \log a_{t_{k}}\right)\right]^{-1 / 2} .
$$

ii) If $\liminf _{k \rightarrow \infty}\left(t_{k+1}-t_{k}\right) / a_{t_{k}}>1$, then

$$
\underset{k \rightarrow \infty}{\limsup } \lambda_{\left(t_{k}, \alpha\right)}\left|W\left(t_{k}+a_{t_{k}}\right)-W\left(t_{k}\right)\right|=\lim \sup _{k \rightarrow \infty} \sup _{0 \leq s \leq a_{t_{k}}} \lambda_{\left(t_{k}, \alpha\right)}\left|W\left(t_{k}+s\right)-W\left(t_{k}\right)\right|=\varepsilon^{*} \quad \text { a.s. , }
$$

where $0 \leq \alpha \leq 1, \varepsilon^{*}=\inf \left\{\gamma>0: \sum_{k}\left(g_{\alpha}\left(t_{k}\right)\right)^{-\gamma^{2}}<\infty\right\}$ and $g_{\alpha}\left(t_{k}\right)=t_{k}\left(\log t_{k}\right)^{\alpha}\left(\log a_{t_{k}}\right)^{1-\alpha} / a_{t_{k}}$.

In this paper the limit theorems on increments of a Wiener process due to [1] are developed to the case of a Gaussian process. This can be considered also as an extension of the results to Gaussian processes obtained in [2]. Throughout this paper, we shall always assume the following statements: Let $\{X(t) ; t \geq 0\}$ be an almost surely continuous Gaussian process with $X(0)=0, E\{X(t)\}=0$ and $E\{X(t+s)-X(t)\}^{2}=\sigma^{2}(s)$, where $\sigma(s)$ is a function of $s \geq 0$. Further we assume that $\sigma(t), t \geq 0$, is a nondecreasing continuous concave, regularly varying function at exponent $\tau(0<\tau<1)$ at $\infty$ (e.g., if $\{X(t) ; t \geq 0\}$ is a standard Wiener process, then $\sigma(t)=\sqrt{t}$ ).

Let $a_{t}(t>0)$ be a nondecreasing function of $t$ with $0<a_{t} \leq t$. For large $t$, let us denote

$$
\beta_{\left(t_{k}, \alpha\right)}=\left[2 \sigma^{2}\left(a_{t_{k}}\right)\left(\log h_{\alpha}(t)\right)\right]^{-1 / 2}
$$

where $0 \leq \alpha \leq 1$ and $h_{\alpha}(t)=t(\log t)^{\alpha}\left(\log a_{t}\right)^{1-\alpha} / a_{t}$ is an increasing function of $t$.

We define two continuous parameter processes $Y_{1}(t)$ and $Y_{2}(t)$ by

$$
Y_{1}(t)=\sup _{0 \leq s \leq a_{t}}|X(t+s)-X(t)|
$$

and

$$
Y_{2}(t)=\left|X\left(t+a_{t}\right)-X(t)\right|
$$

\section{Main Results}

In this section we provide the following two theorems which are the main results. We concern here with the development of the limit theorems of a Wiener process to the case of a Gaussian process under consideration the above given assumptions.

Theorem 1. Let $a_{t}(t>0)$ be a nondecreasing function of $t$ where $0<a_{t} \leq t$ with the nonincreasing function $a_{t} / t$ and let $\left\{t_{k}\right\}$ be any increasing sequence diverging to $\infty$ such that

$$
\limsup _{k \rightarrow \infty}\left(t_{k+1}-t_{k}\right) / a_{t_{k}}<1
$$

then

$$
\limsup _{k \rightarrow \infty} \beta_{\left(t_{k}, \alpha\right)} Y_{1}\left(t_{k}\right)=1 \quad \text { a.s. }
$$

and

$$
\limsup _{k \rightarrow \infty} \beta_{\left(t_{k}, \alpha\right)} Y_{2}\left(t_{k}\right)=1 \quad \text { a.s., }
$$

where $\beta_{\left(t_{k}, \alpha\right)}=\left[2 \sigma^{2}\left(a_{t_{k}}\right)\left(\log h_{\alpha}(t)\right)\right]^{-1 / 2}$.

We note that $\beta_{\left(t_{k}, \alpha\right)} \geq \lambda_{\left(t_{k}, \alpha\right)}$ for large $k$ in case of the Wiener process. It is interesting to compare (1) and (2) with (4) and (5) respectively. 
Theorem 2. Let $a_{t}(t>0)$ be a nondecreasing function of $t$ where $0<a_{t} \leq t$ with the nonincreasing function $a_{t} / t$ and let $\left\{t_{k}\right\}$ be an increasing sequence diverging to $\infty$ such that

$$
\liminf _{k \rightarrow \infty}\left(t_{k+1}-t_{k}\right) / a_{t_{k}}>1,
$$

then

$$
\limsup _{k \rightarrow \infty} \beta_{\left(t_{k}, \alpha\right)} Y_{1}\left(t_{k}\right)=\varepsilon^{* *} \quad \text { a.s. }
$$

and

$$
\limsup _{k \rightarrow \infty} \beta_{\left(t_{k}, \alpha\right)} Y_{2}\left(t_{k}\right)=\varepsilon^{* *} \quad \text { a.s., }
$$

where $0 \leq \alpha \leq 1$ and $\varepsilon^{* *}=\inf \left\{\gamma>0: \sum_{k}\left(h_{\alpha}\left(t_{k}\right)\right)^{-\gamma^{2}}<\infty\right\}$.

\section{Proofs}

In order to prove Theorems 1 and 2, we need to give the following lemmas.

Lemma 1. (See [3]). For any small $\varepsilon^{\prime}>0$ there exists a positive $C_{\varepsilon^{\prime}}$ depending on $\varepsilon^{\prime}$ such that for all $u>0$

$$
P\left\{\sup _{0 \leq s \leq m}\left|\frac{X(t+s)-X(t)}{\sigma(m)}\right|>u\right\} \leq C_{\varepsilon^{\prime}} u \mathrm{e}^{-u^{2} /\left(2+\varepsilon^{\prime}\right)},
$$

where $m$ is any large number and $\{X(t) ; t \geq 0\}$ is defined above.

Lemma 2. (See [4]) Let $\{X(t) ; t \in T\}$ and $\{Y(t) ; t \in T\}$ be centered Gaussian processes such that $E X^{2}(t)=E Y^{2}(t)$ for all $t \in T$ and $E\{X(t) X(s)\} \leq E\{Y(t) Y(s)\}$ for all $s, t \in T$. Then for any real number $u$

$$
P\left\{\sup _{t \in T} X(t) \leq u\right\} \leq P\left\{\sup _{t \in T} Y(t) \leq u\right\} .
$$

Proof of Theorem 1. Firstly, we prove that

$$
\limsup _{k \rightarrow \infty} \beta_{\left(t_{k}, \alpha\right)}\left|Y_{1}\left(t_{k}\right)\right| \leq 1 \quad \text { a.s. }
$$

For any $\left\{t_{k}\right\}$ with the condition (3), we define an increasing sequence $\left\{u_{k}\right\}$ by

$$
0<u_{k}<t_{k} \leq u_{k+1} \text { and } a_{u_{k}}<t_{k+1}-t_{k}, k \geq 1 \text {. }
$$

For instance, let $t_{k}=k^{\beta}$ for some $\beta \geq 1$,

$$
u_{k}=\left(\frac{k}{k+1}\right)^{\beta} t_{k} \text { and } a_{t_{k}}=\left(\frac{k+1}{k+2}\right)^{\beta} t_{k} .
$$

The condition (3) is satisfied, and for large $k, u_{k}<t_{k} \leq u_{k+1}$ and $a_{u_{k}}<a_{t_{k}}<t_{k}$. By Lemma 1, we have, for any small $\varepsilon>0$,

$$
\begin{aligned}
P\left\{\beta_{\left(t_{k}, \alpha\right)} Y_{1}\left(u_{k}\right) \leq 1+\varepsilon\right\} & =P\left\{\sup _{0 \leq s \leq a_{u_{k}}} \frac{X\left(u_{k}+s\right)-X\left(u_{k}\right)}{\sigma\left(a_{u_{k}}\right)} \leq(1+\varepsilon)\left(2 \log h_{\alpha}(t)\right)^{1 / 2}\right\} \\
& \geq 1-2 C_{\varepsilon}\left(h_{\alpha}\left(u_{k}\right)\right)^{-2(1+\varepsilon)^{2} /(2+\varepsilon)} \geq \exp \left(-C^{\prime}\left(h_{\alpha}\left(u_{k}\right)\right)^{-1}\right) \\
& \geq \exp \left(-C^{\prime} /\left(\left(\log u_{k}\right)^{\alpha}\left(\log a_{u_{k}}\right)^{1-\alpha}\right)\right) \\
& \geq \exp \left(-C^{\prime}\left(\log u_{k} / \log a_{u_{k}}\right)^{1-\alpha}\left(1 / \log u_{k}\right)\right) \\
& \geq \exp \left(-C^{\prime}\left(\log u_{k} / \log a_{u_{k}}\right)\left(1 / \log u_{k}\right)\right) \\
& \geq \exp \left(-C^{\prime}\left(\log a_{u_{k}}\right)^{-1}\right)
\end{aligned}
$$


where $k$ is large enough and $C^{\prime}$ is a constant. By the definition of $a_{u_{k}}, \quad S=\sum_{k} \exp \left(-C^{\prime}\left(\log a_{u_{k}}\right)^{-1}\right)=\infty$.

We shall follow the similar proof process as in [5]. Set

$$
S=\sum_{k} \exp \left(-C^{\prime}\left(\log a_{u_{2 k-1}}\right)^{-1}\right)+\sum_{k} \exp \left(-C^{\prime}\left(\log a_{u_{2 k}}\right)^{-1}\right)=S_{1}+S_{2} .
$$

Since $\left\{a_{u_{k}}\right\}$ is an increasing sequence, the fact that $S=\infty$ implies $S_{1}=S_{2}=\infty$. Consider the odd subsequence $\left\{t_{2 k-1}\right\}$ of $\left\{t_{k}\right\}$ and define the sequence of events $\left\{A_{k}\right\}$ in the following form

$$
A_{k}=\left\{\beta_{\left(t_{2 k-1}, \alpha\right)} Y_{1}\left(t_{2 k-1}\right) \leq 1+\varepsilon\right\} .
$$

By (10), for large $k$ we have

$$
P\left(A_{k}\right) \geq \exp \left(-C^{\prime \prime}\left(\log a_{t_{2 k-1}}\right)^{-1}\right)
$$

where $C^{\prime \prime}$ is a constant. From the fact $u_{2 k-1}<t_{2 k-1} \leq u_{2 k}$, it is clear that

$$
P\left(A_{k}\right) \geq \exp \left(-C^{\prime \prime}\left(\log a_{u_{2 k-1}}\right)^{-1}\right) .
$$

Since $S_{1}=\infty$, we get $\sum_{k} P\left(A_{k}\right)=\infty$. Also,

$$
t_{2 k-1}+a_{t_{2 k-1}} \leq u_{2 k}+a_{u_{2 k}}<t_{2 k}+a_{u_{2 k}}=t_{2 k+1} .
$$

Setting

$$
A_{k}^{\prime}=\left\{\sup _{0 \leq s \leq a_{t_{2 k-1}}} \beta_{\left(t_{2 k-1}, \alpha\right)}\left(X\left(t_{2 k-1}+s\right)-X\left(t_{2 k-1}\right)\right) \leq 1+\varepsilon\right\}
$$

and

$$
A_{k}^{\prime \prime}=\left\{\sup _{0 \leq s \leq a_{t_{2 k-1}}} \beta_{\left(t_{2 k-1}, \alpha\right)}\left(X\left(t_{2 k-1}+s\right)-X\left(t_{2 k-1}\right)\right) \geq-1-\varepsilon\right\},
$$

we have

$$
\sum_{k} P\left(A_{k}^{\prime}\right)=\sum_{k} P\left(A_{k}^{\prime \prime}\right)=\infty
$$

Let

$$
X_{1}=\sup _{0 \leq s \leq a_{t_{2 k-1}}}\left(X\left(t_{2 k-1}+s\right)-X\left(t_{2 k-1}\right)\right)=\left(X\left(t_{2 k-1}+s_{1}\right)-X\left(t_{2 k-1}\right)\right),
$$

and

$$
X_{2}=\sup _{0 \leq s \leq a_{t_{2 k+1}}}\left(X\left(t_{2 k+1}+s\right)-X\left(t_{2 k+1}\right)\right)=\left(X\left(t_{2 k+1}+s_{2}\right)-X\left(t_{2 k+1}\right)\right) .
$$

Then, by (11) and the concavity of $\sigma^{2}(t)$ we find that

$$
\begin{aligned}
\operatorname{Cov}\left(X_{1}, X_{2}\right) & =E\left\{X\left(t_{2 k+1}+s_{2}\right) X\left(t_{2 k-1}+s_{1}\right)\right\}-E\left\{X\left(t_{2 k+1}+s_{2}\right) X\left(t_{2 k-1}\right)\right\} \\
& -E\left\{X\left(t_{2 k+1}\right) X\left(t_{2 k-1}+s_{1}\right)\right\}+E\left\{X\left(t_{2 k+1}\right) X\left(t_{2 k-1}\right)\right\} \\
& =1 / 2\left\{\sigma^{2}\left(t_{2 k+1}-t_{2 k-1}+s_{2}\right)-\sigma^{2}\left(t_{2 k+1}-t_{2 k-1}+s_{2}-s_{1}\right)\right\} \\
& -1 / 2\left\{\sigma^{2}\left(t_{2 k+1}-t_{2 k-1}\right)-\sigma^{2}\left(t_{2 k+1}-t_{2 k-1}-s_{1}\right)\right\} .
\end{aligned}
$$

This implies that $\operatorname{Cov}\left(X_{1}, X_{2}\right) \leq 0$. Using Lemma 2, we obtain

$$
P\left(A_{k}^{\prime} \cap A_{1}^{\prime}\right) \leq P\left(A_{k}^{\prime}\right) P\left(A_{1}^{\prime}\right) \text { and } P\left(A_{k}^{\prime \prime} \cap A_{1}^{\prime \prime}\right) \leq P\left(A_{k}^{\prime \prime}\right) P\left(A_{l}^{\prime \prime}\right)
$$

where $k \neq l$. It follows from the Borel-Cantelli lemma that 


$$
-1-\varepsilon \leq \limsup _{k \rightarrow \infty} \sup _{0 \leq s \leq a_{t_{2 k-1}}} \beta_{\left(t_{2 k-1}, \alpha\right)}\left(X\left(t_{2 k-1}+s\right)-X\left(t_{2 k-1}\right)\right) \leq 1+\varepsilon \text {, a.s. }
$$

Also, the same result for the even subsequence $\left\{t_{2 k}\right\}$ of $\left\{t_{k}\right\}$ is easily obtained. Therefore we have (9). To finish the proof of Theorem 1, we need to prove

$$
\limsup _{k \rightarrow \infty} \beta_{\left(t_{k}, \alpha\right)} Y_{2}\left(t_{k}\right) \geq 1 \quad \text { a.s. }
$$

The proof of (12) is similar to the provided proof in [1]. Thus the proof of Theorem 1 is complete.

Proof of Theorem 2. Firstly, we prove that

$$
\limsup _{k \rightarrow \infty} \beta_{\left(t_{k}, \alpha\right)} Y_{1}\left(t_{k}\right) \leq \varepsilon^{* *} \quad \text { a.s. }
$$

According to Lemma 1 , we have

$$
\begin{aligned}
& P\left\{\beta_{\left(t_{k}, \alpha\right)} Y_{1}\left(t_{k}\right) \geq \varepsilon^{* *}+\right.\varepsilon\} \\
&=P\left\{\sup _{0 \leq s \leq a_{t_{k}}} \frac{X\left(t_{k}+s\right)-X\left(t_{k}\right)}{\sigma\left(a_{t_{k}}\right)} \geq\left(\varepsilon^{* *}+\varepsilon\right)\left(2 \log h_{\alpha}\left(t_{k}\right)\right)^{1 / 2}\right\} \\
& \leq 2 C_{\varepsilon}\left(h_{\alpha}\left(t_{k}\right)\right)^{-2\left(\varepsilon^{* *}+\varepsilon\right)^{2} /(2+\varepsilon)} \\
& \leq 2 C_{\varepsilon}\left(h_{\alpha}\left(t_{k}\right)\right)^{-2\left(\varepsilon^{* *}+\varepsilon_{1}\right)^{2}}
\end{aligned}
$$

provided $k$ is large enough, where $\varepsilon>0$ and $0<\varepsilon_{1}<\varepsilon^{3 / 2}$.

From the definition of $\varepsilon^{* * *}$, it follows that

$$
\sum_{k} P\left\{\beta_{\left(t_{k}, \alpha\right)} Y_{1}\left(t_{k}\right) \geq \varepsilon^{* *}+\varepsilon\right\}<\infty .
$$

Thus, (13) is immediate by using Borel Cantelli lemma.

To finish the proof of Theorem 2 we need to prove

$$
\limsup _{k \rightarrow \infty} \beta_{\left(t_{k}, \alpha\right)}\left(X\left(t_{k}+a_{t_{k}}\right)-X\left(a_{t_{k}}\right)\right) \geq \varepsilon^{* * *} \text {, a.s. }
$$

Let

$$
\Phi(u)=\frac{1}{\sqrt{2 \pi}} \int_{u}^{+\infty} \mathrm{e}^{-x^{2} / 2} \mathrm{~d} x, \quad u \geq 0 .
$$

Using the well known probability inequality

$$
\frac{1}{\sqrt{2 \pi}(u+1)} \mathrm{e}^{-u^{2} / 2} \leq \Phi(u) \leq \frac{4}{3} \frac{1}{\sqrt{2 \pi}(u+1)} \mathrm{e}^{-u^{2} / 2}, \quad u \geq 0
$$

(see [6]), one can find positive constants $C$ and $K$ such that, for all $k \geq K$,

$$
\begin{aligned}
P\left(B_{k}\right) & =P\left\{\frac{X\left(t_{k}+a_{t_{k}}\right)-X\left(a_{t_{k}}\right)}{\sigma\left(a_{t_{k}}\right)} \geq\left(\varepsilon^{* *}-\varepsilon\right)\left(2 \log h_{\alpha}\left(t_{k}\right)\right)^{1 / 2}\right\} \\
& \geq \frac{1}{\sqrt{2 \pi}}\left\{\left(\varepsilon^{* *}-\varepsilon\right)\left(2 \log h_{\alpha}\left(t_{k}\right)\right)^{1 / 2}+1\right\}^{-1}\left(h_{\alpha}\left(t_{k}\right)\right)^{-\left(\varepsilon^{* *}-\varepsilon\right)^{2}} \\
& \geq C\left(h_{\alpha}\left(t_{k}\right)\right)^{-\left(\varepsilon^{* *}-\varepsilon^{\prime}\right)^{2}}
\end{aligned}
$$

where $0<\varepsilon^{\prime}<\varepsilon<\varepsilon^{* * *}$ and $B_{k}=\left\{\beta_{\left(t_{k}, \alpha\right)}\left(X\left(t_{k}+a_{t_{k}}\right)-X\left(a_{t_{k}}\right)\right) \geq\left(\varepsilon^{* *}-\varepsilon\right)\right\}$. By the definition of $\varepsilon^{* * *}$, we have $\sum_{k} P\left(B_{k}\right)=\infty$.

The condition (6) implies that there exists $K>0$ such that $t_{k+1} \geq t_{k}+a_{t_{k}}$ for all $k \geq K$. So, using Lemma 2 
and the concavity of $\sigma^{2}(t)$, we obtain

$$
P\left(B_{k} \cap B_{l}\right) \leq P\left(B_{k}\right) P\left(B_{l}\right),
$$

where $k \neq l$ and Borel-Cantelli lemma implies (14). If $\varepsilon^{* *}=0$, then Theorem 2 is immediate. Thus the proof of Theorem 2 is complete.

\section{Some Results for Partial Sums of Stationary Gaussian Sequence}

In this section we obtain similar results as Theorems 1 and 2 for the case of partial sums of a stationary Gaussian sequence. Let $\left\{X_{n}\right\}$ be a stationary Gaussian sequence with $X_{0}=0, E\left\{X_{1}\right\}=0, E\left\{X_{1}^{2}\right\}=1$ and $E\left\{X_{1} X_{1+n}\right\} \leq 0$ for all $n=1,2, \cdots$. We define $S(n)=\sum_{i=1}^{n} X_{i}$ with $S(0)=0$ and set $\sigma^{2}(n)=E\left\{S^{2}(n)\right\}$. Assume that $\sigma(n)$ can be extended to a continuous function $\sigma(t)$ with $t>0$ which is nondecreasing and regularly varying with exponent $\tau(0<\tau<1)$ at $\infty$. Suppose that $\left\{a_{n}\right\}$ is a nondecreasing sequence of positive integers such that $0 \leq a_{n} \leq n$. For large $n$, we define

$$
\beta_{(n, \alpha)}=\left[2 \sigma^{2}\left(a_{n}\right)\left(\log h_{\alpha}(n)\right)\right]^{-1 / 2},
$$

where $0 \leq \alpha \leq 1$ and $h_{\alpha}(n)=n(\log n)^{\alpha}\left(\log a_{n}\right)^{1-\alpha} / a_{n}$ is an increasing function of $n$ and also we define discrete time parameter processes by

$$
Y_{1}\left(n_{k}\right)=\max _{0 \leq j \leq a_{n_{k}}}\left|S\left(n_{k}+j\right)-S\left(n_{k}\right)\right|
$$

and

$$
Y_{1}\left(n_{k}\right)=\max _{0 \leq j \leq a_{n_{k}}}\left|S\left(n_{k}+a_{n_{k}}\right)-S\left(n_{k}\right)\right|,
$$

respectively, where $\left\{n_{k}\right\}$ is an increasing sequence of positive integers diverging to $\infty$. By the same way as in the proofs of Theorems 1 and 2, we obtain the following results.

Theorem 3. Under the above statements of $\left\{X_{n}\right\}, \beta_{(n, \alpha)}$ and $Y_{i}\left(n_{k}\right), i=1,2$, for $0 \leq \alpha \leq 1$ we have the following:

i) If $\lim \sup \left(n_{k+1}-n_{k}\right) / a_{n_{k}}<1$, then

$$
\limsup _{k \rightarrow \infty} \beta_{\left(n_{k}, \alpha\right)} Y_{i}\left(n_{k}\right)=1 \quad \text { a.s., } i=1,2 \text {. }
$$

ii) If $\lim \inf \left(n_{k+1}-n_{k}\right) / a_{n_{k}}>1$, then

$$
\limsup _{k \rightarrow \infty} \beta_{\left(n_{k}, \alpha\right)} Y_{i}\left(n_{k}\right) \leq \varepsilon^{* *} \quad \text { a.s., } i=1,2,
$$

where

$$
\varepsilon^{* *}=\inf \left\{\gamma>0: \sum_{k}\left(h_{\alpha}\left(n_{k}\right)\right)^{-\gamma^{2}}<\infty\right\} .
$$

Example. Let $\{X(t) ; 0 \leq t<\infty\}$ be a fractional Brownian motion with the covariance function $E\{X(t) X(s)\}=\left\{|t|^{\tau}+|s|^{\tau}-|t-s|^{\tau}\right\} / 2,0<\tau<1$. Then

$$
E\{X(t)-S(s)\}^{2}=|t-s|^{\tau}
$$

Define random variables

$$
\begin{gathered}
X_{0}=0, \\
X_{n}=X_{(n)}-X_{(n-1)}, n=1,2, \cdots, \\
S(n)=\sum_{i=1}^{n} X_{i} \text { and } S(0)=0 .
\end{gathered}
$$

Then 


$$
\sigma^{2}(n)=E\left\{S^{2}(n)\right\}=E\left\{X^{2}(n)\right\}=n^{\tau}
$$

and $\left\{X_{n} ; n=1,2, \cdots\right\}$ is a stationary Gaussian sequence with $E\left\{X_{1}\right\}=0, E\left\{X_{1}^{2}\right\}=1$ and $E\left\{\left(X_{1} X_{1+n}\right)\right\} \leq 0$ for all $n=1,2, \cdots$. So we have Theorem 3 .

In particular if $\tau=1 / 2$, then $\left\{X_{n} ; n=1,2, \cdots\right\}$ is an i.i.d. Gaussian sequence with $E\left\{X_{1}\right\}=0$ and $E\left\{X_{1}^{2}\right\}=1$.

\section{Conclusion}

In this paper, we developed some limit theorems on increments of a Wiener process to the case of a Gaussian process. Moreover, we obtained similar results of these limit theorems for the case of partial sums of a stationary Gaussian sequence. Some obtained results can be considered as extensions of some previous given results to Gaussian processes.

\section{References}

[1] Bahram, A. (2014) Convergence of the Increments of a Wiener Process. Acta Mathematica Universitatis Comenianae, 83, 113-118.

[2] Hwang, K.S., Choi, Y.K. and Jung, J.S. (1997) On Superior Limits for the Increments of Gaussian Processes. Statistics and Probability Letters, 35, 289-296. http://dx.doi.org/10.1016/S0167-7152(97)00025-4

[3] Choi, Y.K. (1991) Erdös-Réyi Type Laws Applied to Gaussian Process. Journal of Mathematics of Kyoto University, 31, 191-217.

[4] Slepian, D. (1962) The One-Sided Barrier Problem for Gaussian Noise. Bell System Technical Journal, 41, 463-501. http://dx.doi.org/10.1002/j.1538-7305.1962.tb02419.x

[5] Vasudeva, R. and Savitha, S. (1993) On the Increments of Weiner Process-A Look through Subsequences. Stochastic Processes and Their Applications, 47, 153-158. http://dx.doi.org/10.1016/0304-4149(93)90101-9

[6] Fernique, X. (1975) Evaluations of Processus Gaussian Composes. Probability in Banach Spaces. Lecture Notes in Mathematics, 526, 67-83. 